\title{
Influence of location, number and shape of corrugations in slider bearings
}

\author{
B. P. Huynh S. Loe*
}

(Received 8 August 2003; 19 September 2004)

\begin{abstract}
The effects of location and shape of a localised corrugation on the performance of a fixed inclined slider bearing are investigated numerically, using a finite element method. It is shown that normal force increases uniformly as the corrugation is relocated downstream in the bearing gap, from being less than that of the smooth walled case to being higher, while flow rate decreases, also uniformly. Corrugation with the shape of rectangle waves produce largest changes in pressure distribution, normal force and flow rate, relative to the smooth walled case. This is followed by sine-wave corrugation, whereas triangle-wave corrugation produces smallest changes. The number of corrugation waves produces similar, but weaker, changes in normal force and flow rate, to the corrugation volume, when this is used as a parameter characterizing corrugation shape.
\end{abstract}

*Faculty of Engineering, University of Technology, Sydney, Broadway, NSW 2007, Australia. mailto:Phuoc. Huynh@uts. edu.au

See http://anziamj.austms.org.au/V45/CTAC2003/Huyn/home.html for this article, (c) Austral. Mathematical Soc. 2004. Published October 1, 2004. ISSN 1446-8735 


\section{Contents}

1 Introduction

C1018

2 Mathematical model and numerical method

C1019

3 Results and discussion

C1024

3.1 Effects of corrugation shape . . . . . . . . . . . C1026

3.2 Effects of corrugation location . . . . . . . . . . . C1031

3.3 Effects of the number of corrugation waves . . . . . . C C1031

4 Conclusions

C1034

References

C1034

\section{Introduction}

Influence of surface corrugation on the performance of slider bearings has attracted the attention of many investigators $[1,2,3,4,5,6,7,8,9]$. However, in these works, invariably corrugation extends over the full length (in the direction of sliding motion) of the bearing gap. On the other hand, there can be many situations where corrugation occurs not over the whole bearing, but rather in some sections only. This could happen intentionally, or as a result of manufacturing error, or some non-uniform flow pattern causing localized erosion and corrugation, particularly if solid foreign particles are present. In other words, corrugation of limited extent may arise.

In an effort to provide further understanding of lubrication flow in bearings, this work considers the effects of corrugation of different shape, and of very limited extent, on the performance of fixed-incline slider bearings, using viscous fluids of a Newtonian type for lubricant. A finite element numerical method is used for computation of the flow under non-isothermal 
conditions. Also, unlike many previous works where often approximations of a Reynolds type $[3,4,5,6,9]$, or to a lesser extent, approximations of a Stokes type $[7,8]$ have been used, no simplifying assumptions will be made in the present study.

\section{Mathematical model and numerical method}

The modelled flow domain, which is two-dimensional and corresponds to a slider bearing's fluid film (or gap) with rectangular coordinates $x$ and $y$, is shown diagrammatically in Figure 1. In this figure the lower base surface is a smooth plane which slides in the $x$-direction at constant speed $u_{b}$. The gap's upper, inclined surface is stationary and has a corrugated section. In the $x$-direction, the flow domain is bounded at coordinates $x_{i}$ at the inlet, and $x_{o}$ at outlet. The flow domain's inlet height is $h_{i}$, and its outlet height $h_{o}$.

The corrugated section of the stationary, inclined surface starts with an $x$-coordinate $x_{s}$, and extends over a distance $d$. The gap height corresponding to a smooth inclined surface at $x_{s}$ is $h_{s}$.

Corrugation is in the form of regular waves of amplitude $a$, and with wave shape of triangle, sine function and rectangle. A whole number $n$ of wave cycles cover the corrugated section. Here, only small values for $n$ have been considered; namely only $n=1,2$ and 3 are used, with $n=0$ being the special case of smooth wall.

Thus in terms of $x$-coordinates, the inclined surface is smooth from $x_{i}$ to $x_{s}$, corrugated from $x_{s}$ to $\left(x_{s}+d\right)$ then smooth again to $x_{w}$. This inclined surface is followed immediately by a very short section with parallel lower and upper walls between $x_{w}$ and $x_{o}$. This short section with uniform height is incorporated in the model to help with the imposition of correct veloc- 


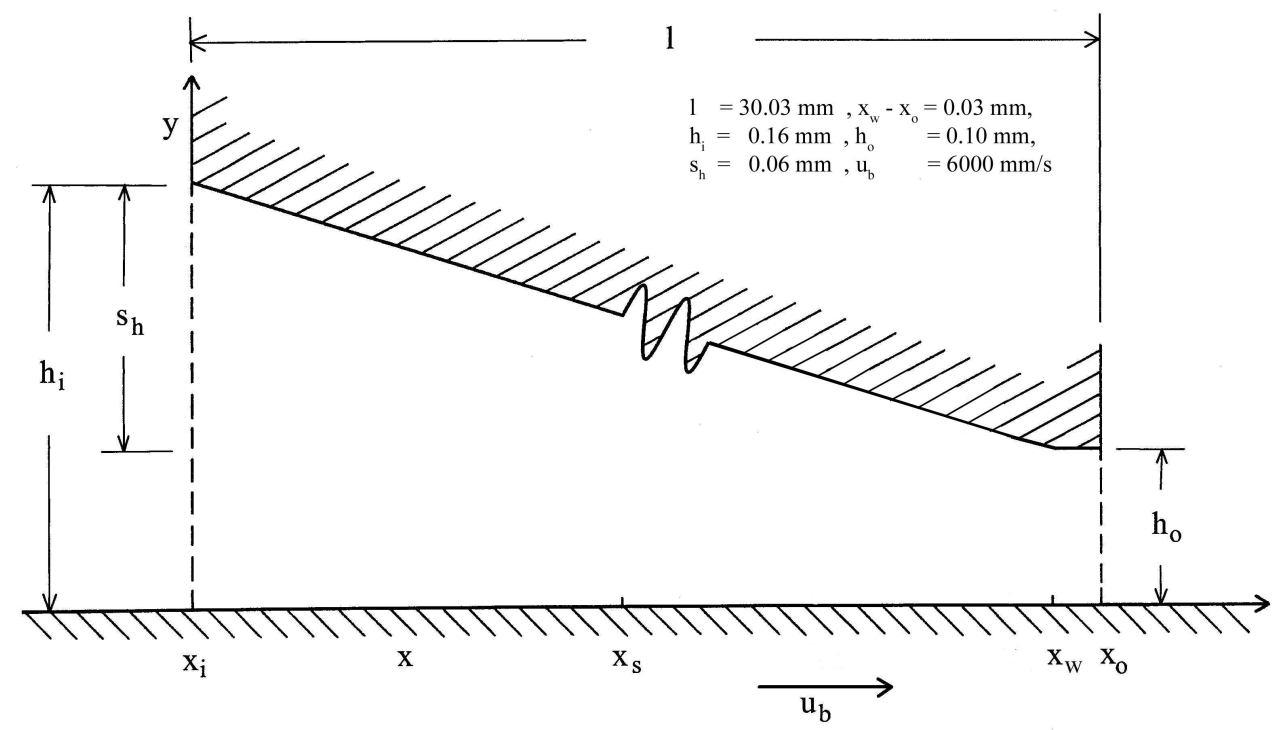

FiguRE 1: Slider bearing geometry with corrugated inclined surface. See Section 2 for explanation of symbols. 
ity boundary condition at outlet of the flow domain, as explained below in relation to boundary conditions.

In this work, the standard units used will be mm, g, s and ${ }^{\circ} \mathrm{C}$. Referring to Figure 1, the following dimensions and coordinates are used: $d=1 \mathrm{~mm}$, length of corrugated section; $h_{i}=0.16 \mathrm{~mm} ; h_{o}=0.10 \mathrm{~mm} ; s_{h}=h_{i}-h_{o}=$ $0.06 \mathrm{~mm}$, shoulder height; $x_{i}=0 ; x_{w}=30 \mathrm{~mm} ; x_{o}=30.03 \mathrm{~mm} ; l=x_{o}-x_{i}=$ $30.03 \mathrm{~mm}$, bearing length; $y$-coordinate is measured from the sliding base plane; $u_{b}=6000 \mathrm{~mm} / \mathrm{s} ; x_{s}, h_{s}, a$ and $n$ are as defined above; and corrugation wavelength is $\lambda=d / n$.

From these dimensions, the mean angle between the inclined and sliding surfaces is $\tan ^{-1}(0.06 / 30)=0.002 \mathrm{rad}$.

Fluid properties, which correspond to an oil, have been adopted from Frêne et al. [10]. The following properties are assumed to be constant: Density $\rho=8.60 \times 10^{-4} \mathrm{~g} / \mathrm{mm}^{3}\left(860 \mathrm{~kg} / \mathrm{m}^{3}\right)$; Specific heat capacity $c=2 \times$ $10^{9} \mathrm{~mm}^{2} / \mathrm{s}^{2}{ }^{\circ} \mathrm{C}\left(2000 \mathrm{~J} / \mathrm{kg}^{\circ} \mathrm{C}\right)$; Thermal conductivity $k=1.3 \times 10^{5} \mathrm{~g} \mathrm{~mm} / \mathrm{s}^{3}{ }^{\circ} \mathrm{C}$ $\left(0.13 \mathrm{~W} / \mathrm{m}^{\circ} \mathrm{C}\right)$.

Viscosity $\mu$ varies exponentially with temperature $T$ (in ${ }^{\circ} \mathrm{C}$ ) according to the relation [10] $\mu=\mu_{o} e^{-\alpha(T-\beta)}$, where $\beta=30^{\circ} \mathrm{C}$ is the fluid's "base" temperature, $\mu_{o}=0.03934 \mathrm{~g} / \mathrm{mms}$ the fluid viscosity at base temperature (note: $1 \mathrm{~g} / \mathrm{mms}=1 \mathrm{Pas}$ ), and $\alpha=0.034\left({ }^{\circ} \mathrm{C}\right)^{-1}$ fluid viscosity's exponential coefficient.

Note that when it is desired to neglect the effects of temperature upon the flow field, $\alpha$ is set to 0 , and the corresponding case is called "isothermal" here. In fact, as will be explained below in Section 3, all computational results are presented for this "isothermal" case only. However, the numerical procedure has been developed for the more general, non-isothermal situation.

The mathematical model used is that for a steady, two-dimensional flow of incompressible, Newtonian fluids without body forces. 
The corresponding governing equations are those of conservation of mass and momentum, and balance of energy, in full. In the usual notation, these equations in rectangular coordinates are [11]: Conservation of mass,

$$
\frac{\partial u}{\partial x}+\frac{\partial v}{\partial y}=0 ;
$$

Conservation of momentum,

$$
\begin{aligned}
& \rho\left(u \frac{\partial u}{\partial x}+v \frac{\partial u}{\partial y}\right)=-\frac{\partial p}{\partial x}+\mu\left(\frac{\partial^{2} u}{\partial x^{2}}+\frac{\partial^{2} u}{\partial y^{2}}\right) \\
& \rho\left(u \frac{\partial v}{\partial x}+v \frac{\partial v}{\partial y}\right)=-\frac{\partial p}{\partial y}+\mu\left(\frac{\partial^{2} v}{\partial x^{2}}+\frac{\partial^{2} v}{\partial y^{2}}\right)
\end{aligned}
$$

Balance of energy,

$$
\begin{aligned}
& \rho c\left(u \frac{\partial T}{\partial x}+v \frac{\partial T}{\partial y}\right)=k\left(\frac{\partial^{2} T}{\partial x^{2}}+\frac{\partial^{2} T}{\partial y^{2}}\right) \\
& +2 \mu\left[\left(\frac{\partial u}{\partial x}\right)^{2}+\left(\frac{\partial v}{\partial y}\right)^{2}+\frac{1}{2}\left(\frac{\partial u}{\partial y}+\frac{\partial v}{\partial x}\right)^{2}\right]
\end{aligned}
$$

where $u$ and $v$ are respectively the velocity components in $x$ and $y$ direction, $p$ pressure, and $T$ temperature. Note that, as mentioned above, the sliding motion is in the $x$ direction, and $y$ is thus the "normal" direction.

Note also that for a Newtonian fluid and in Cartesian component form, the stress $t_{i j}$ is related to pressure and strain rates by

$$
t_{i j}=-p \delta_{i j}+\mu\left(\frac{\partial v_{i}}{\partial x_{j}}+\frac{\partial v_{j}}{\partial x_{i}}\right),
$$

where $v_{i}$ is the velocity in the $x_{i}$ direction, and $\delta_{i j}$ the Kronecker delta.

Boundary Conditions Referring to Figure 1 and using standard notation, the following boundary conditions are used: 
1. At inlet to the flow domain $\left(x=x_{i}\right)$ : a profile of the normal (ydirection) velocity $v$ is prescribed according to the theoretical formula [12]

$$
\frac{v l}{u_{b} s_{h}}=\left(\frac{Y^{2}}{H_{i}^{4}}\right)\left(Y-H_{i}\right)\left(3 H_{p}-2 H_{i}\right),
$$

where $H_{o}=h_{o} / s_{h}, Y=y / s_{h}, H_{i}=h_{i} / s_{h}$, and $H_{p}=2 H_{o}\left(1+H_{o}\right) /(1+$ $\left.2 H_{o}\right)$. Using the parameter values appropriate to this work, we have $Y=y / 0.06, H_{i}=1.6667, H_{o}=2.6667, H_{p}=2.0513$; and $v$ is thus $v=9.0052 \times 10^{-3} u_{b} y^{2}[(y / 0.06)-2.6667]$.

Also temperature is uniform and set at "base" value: $T=30^{\circ} \mathrm{C}$

2. On the stationary, inclined upper surface: non-slip condition and constant temperature at "base" value $u=v=0, T=30^{\circ} \mathrm{C}$. Note that, as mentioned above, this inclined surface has a corrugated section starting at $x_{s}$

3. At exit of the considered flow domain $\left(x=x_{o}\right)$ : zero $y$-direction velocity and zero normal stress in $x$-direction $v=0, t_{x x}=0$. Note that normal (y-direction) velocity $v$ is zero thanks to the upper and lower bounding surfaces being parallel, as shown in Figure 1.

No thermal boundary condition is imposed on this end, thus the solution attempts to make $\partial T / \partial z=0$ here.

4. On the sliding base surface: $x$-direction velocity equal to the sliding speed and zero velocity in $y$-direction; and constant temperature at "base" value $u=u_{b} ; v=0 ; T=30^{\circ} \mathrm{C}$.

A finite element scheme based on the Galerkin discretisation procedure is used. The numerical method used has been amply verified before, with nontrivial problems in the areas of extrusion, wire-coating, and free convection having been solved $[13,14,15]$, in addition to simpler problems for which analytical solutions are known. With the present problem, good agreement 
with available theoretical solutions [12] has been obtained; this is presented and discussed in the Results and Discussion section below.

Grid patterns of 10 quadrilateral elements in the normal $(y)$ direction by 243 elements in the sliding $(x)$ direction $(11 \times 244$ grid points $)$, and $15 \times 212$ are used. Figure 2 shows an example of a grid pattern used.

Grid convergence tests have been carried out to ascertain the adequacy of the patterns used.

Numerical convergence has been ensured to be excellent. Convergence is verified using the following measure of variation covering the whole flow field $\epsilon=\left|w_{k+1}-w_{k}\right| /\left(1+\left|w_{k+1}\right|\right)$, where $w_{k}$ is the value of the parameter $w$ after the $k$ th iteration; the parameters considered are the two velocity components, pressure and temperature. Here we have followed Gill el al. [16] who suggest an analogous measure of error.

In this work, $\epsilon$ is less than $10^{-7}$ at convergence.

Computation is done on a Sun Enterprise 3000 machine, running a UNIX operating system. Double precision (64 bits) is used throughout.

\section{Results and discussion}

Computation has been performed with a sliding base speed $u_{b}=6000 \mathrm{~mm} / \mathrm{s}$. Corrugation is in the form of regular waves. Three wave patterns, namely triangular, sinusoidal and rectangular, have been considered. Computation is also done for the smooth walled case which is used as a reference. Other changing parameters are the corrugated section's location (indicated by its starting location $x_{s}$ ), number of cycles $n$ and amplitude $a$ of the corrugation's waves. Corrugated section length is kept constant at $1 \mathrm{~mm}$.

In all computational results presented below, the viscosity's exponential 


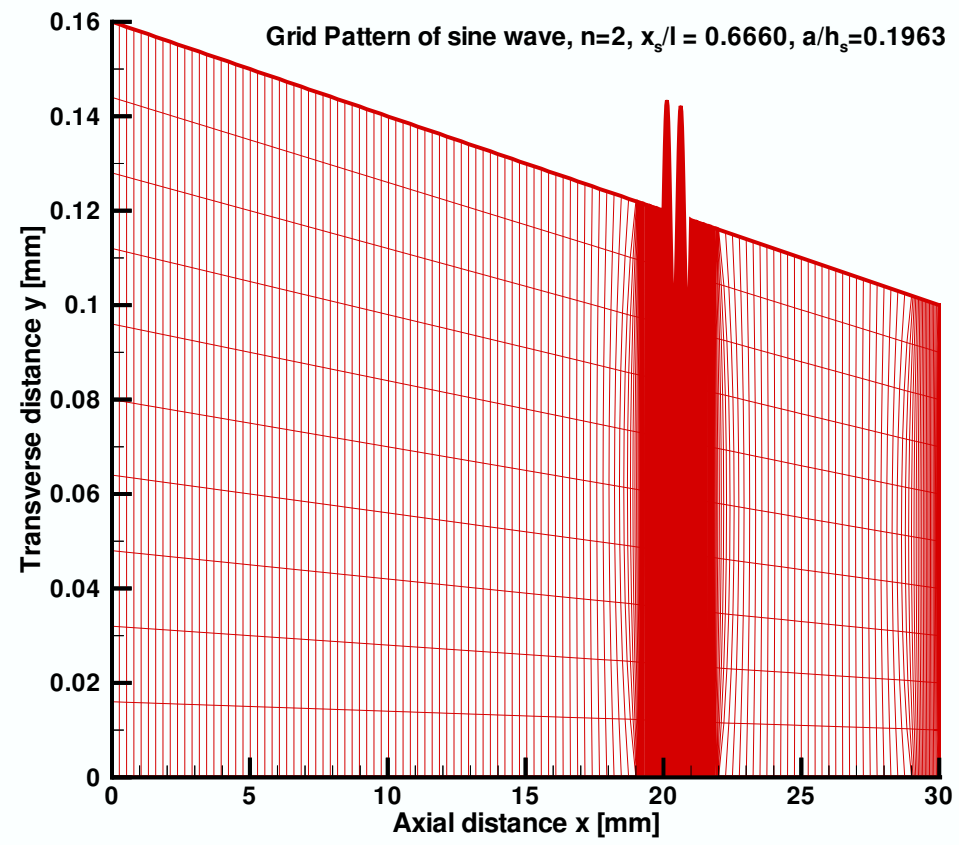

FIGURE 2: Grid pattern of a representative case. 
TABLE 1: Smooth walled, isothermal case; $u_{b}=6000 \mathrm{~mm} / \mathrm{s}$

\begin{tabular}{|c|cc|}
\hline & Theoretical & Computational \\
\hline Maximum pressure, $p_{\max }[\mathrm{Pa}]$ & $1.532 \times 10^{5}$ & $1.538 \times 10^{5}$ \\
$x$-coordinate of $p_{\max }, x_{\max }[\mathrm{mm}]$ & 18.48 & 18.46 \\
Normal force per unit width, $F[\mathrm{mN} / \mathrm{mm}]$ & $3.000 \times 10^{6}$ & $3.011 \times 10^{6}$ \\
Flow rate per unit width, $Q\left[\mathrm{~mm}^{2} / \mathrm{s}\right]$ & 369.2 & 368.7 \\
\hline
\end{tabular}

coefficient $\alpha$ is kept at zero. Temperature change thus has no effects on the flow field. Computation was also performed with $\alpha=0.034\left({ }^{\circ} \mathrm{C}\right)^{-1}$, but the corresponding temperature change is very small, and thermal effects have been seen to be negligible.

It is noted that with $u_{b}=6000 \mathrm{~mm} / \mathrm{s}$, the modified Reynolds number $\operatorname{Re}_{m}=\rho u_{b}\left(h_{o}\right)^{2} / \mu_{o} l$ is 0.044 [12]. However, if the conventional definition $\operatorname{Re}=\rho u_{b} h_{i} / \mu_{o}$ is used for Reynolds number, then the bulk flow's value is quite high, being 21 .

Comparison between computational results and theoretical predictions [12] for the reference smooth walled, isothermal case has been carried out, and the agreement is very good. This is shown in Table 1. The agreement further reinforces confidence in the numerical method used.

\subsection{Effects of corrugation shape}

Three corrugation shapes, namely triangular, sinusoidal and rectangular, have been considered, and their effects on pressure distribution, load carrying capacity and flow rate, have been compared. Figure 3 shows the effects of corrugation shape on pressure distribution along the stationary, inclined surface for representative cases with corrugations located close to the gap exit, at $x_{s}=28.5 \mathrm{~mm}$ or $x_{s} / l=0.9491$. The figure shows that relative to the reference smooth walled case, an increase in pressure is produced with 
all three shapes. But corrugations with shape of rectangle waves produce a much larger increase than the other two shapes; and triangular corrugations result in the smallest change.

Integration of the pressure distribution along the inclined surface gives normal force, which is a bearing's load supporting capacity. Influence of corrugation shape on normal force is shown in Figure 4. It can be seen that rectangle waves produce the largest change in the normal force relative to the smooth walled situation, followed by sinusoidal corrugation. Triangular waves result in the smallest change.

Figure 5 shows change in flow rate with respect of the smooth walled case. It can be seen that all corrugations result in reductions in flow rate (negative change). And in similarity with the normal force, rectangle waves produce largest change (most negative) in flow rate, whereas the least change occurs with triangle waves.

Note that in the two Figure 4 and 5, one horizontal axis has units of (Volume of corrugation waves)/(Volume of triangular corrugation waves). For waves with the same amplitude $a$ and wavelength $\lambda$, the ratio of volumes of corrugation (per unit depth, in the direction perpendicular to the page) between a sine wave and a triangle wave is (considering only a half wave cycle)

$$
\frac{\int_{0}^{\lambda / 2} a \sin (2 \pi x / \lambda) d x}{(1 / 2)(a \times \lambda / 2)}=1.273 .
$$

On the other hand the (Volume of a rectangle wave)/(Volume of a triangle wave $)=(a \times \lambda / 2) /[(1 / 2)(a \times \lambda / 2)]=2$. Thus Figures 4 and 5 also indicate that corrugation with a larger volume gives rise to larger changes in both normal force and flow rate, relative to the smooth walled case. 


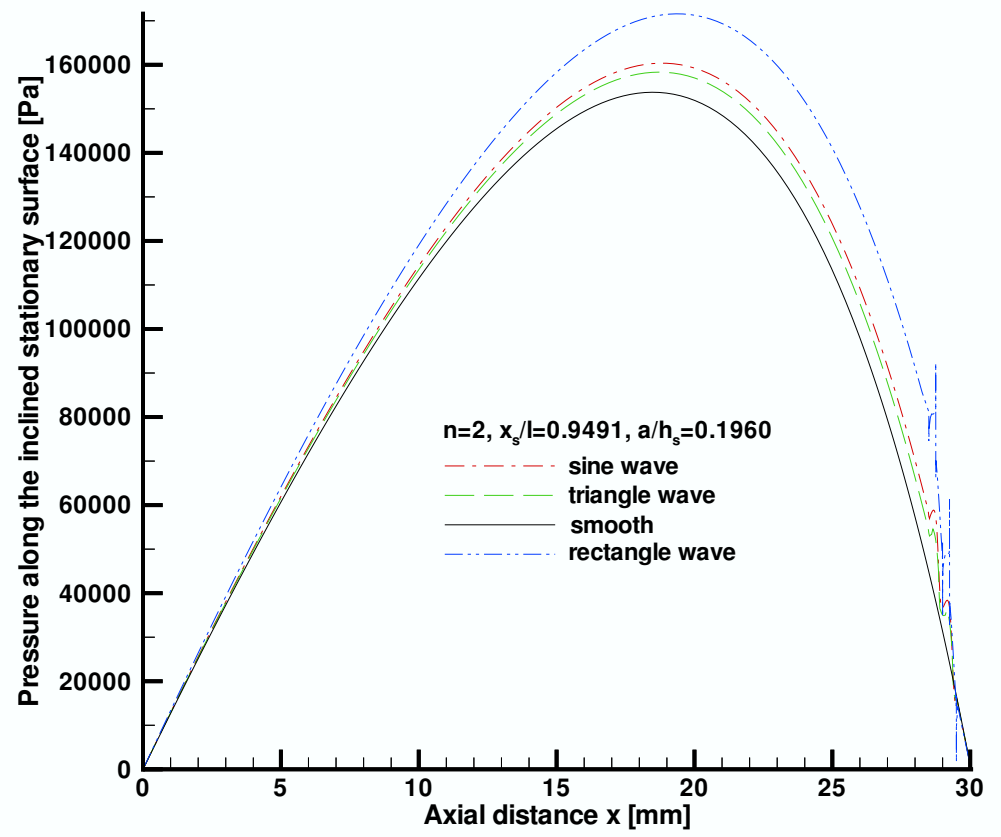

FigURE 3: Effects of corrugation shape on pressure distribution. 


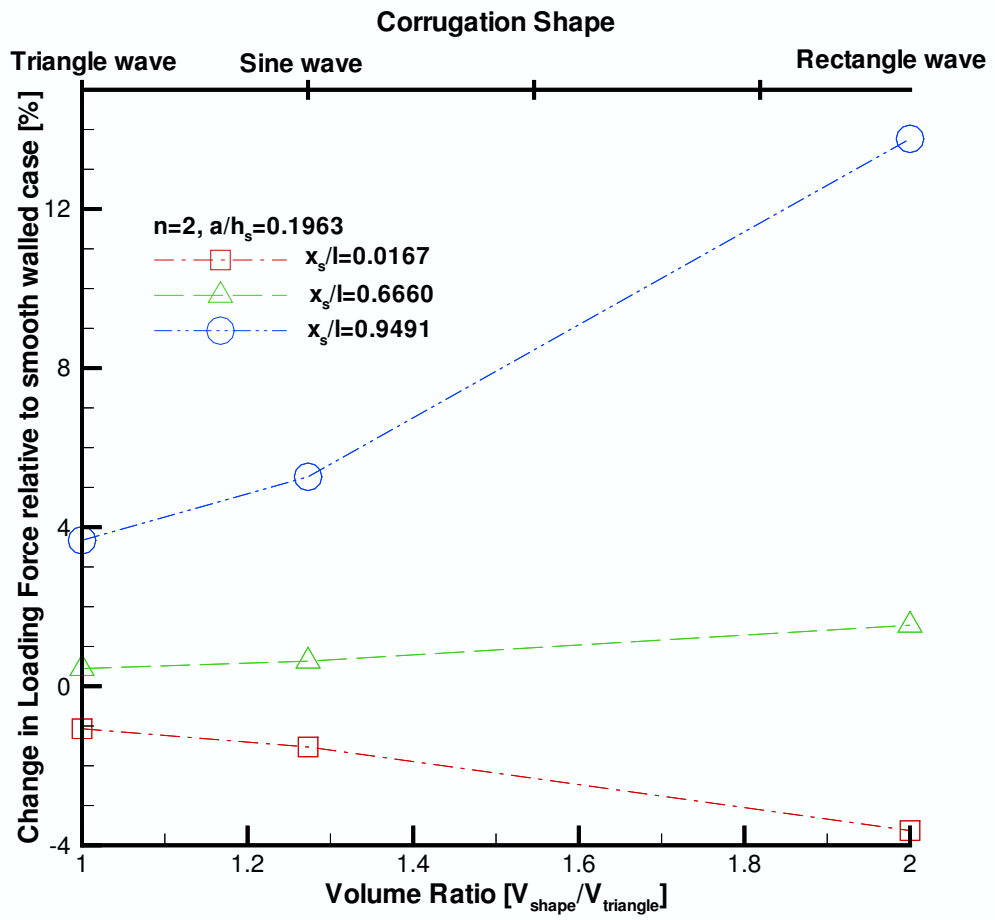

FigURE 4: Effects of corrugation shape and location on normal load. 


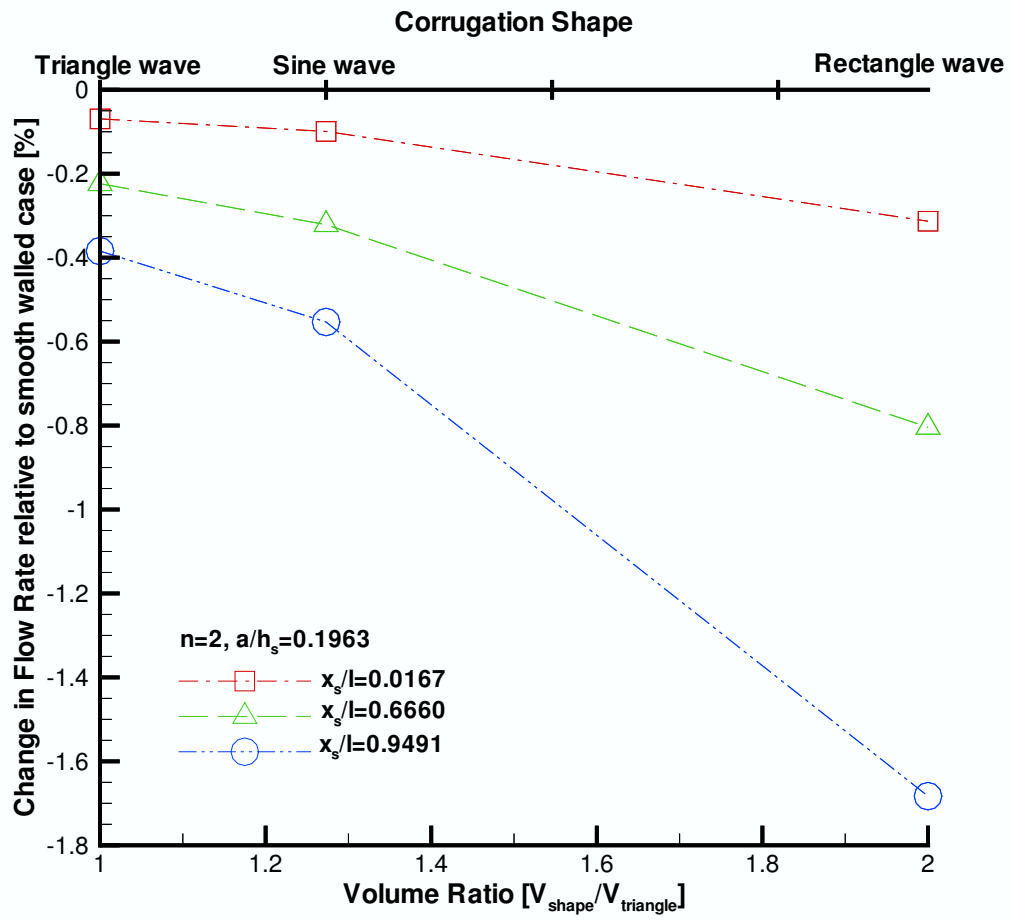

FiguRE 5: Effects of corrugation shape and location on flow rate. 


\subsection{Effects of corrugation location}

Corrugation location is primarily indicated by the corrugated section's starting position whose $x$-coordinate is $x_{s}$. Figure 4 shows that as the corrugations are relocated further downstream, from being near the gap inlet to being near its outlet, normal force increases uniformly, from being lower than the smooth walled case (negative change) to being higher (positive change).

On the other hand, as the corrugations are relocated further downstream toward the gap exit, flow rate decreases, also uniformly, making the already negative change relative to the smooth walled case more pronounced. This is shown in Figure 5.

The enhancing effects of corrugations' downstream location on normal force, and its depressing effects on flow rate, can further be seen in Figures 6 and 7. These figures also show the expected reinforcing influence of corrugation amplitude $a$, in the sense that a larger $a$ value gives rise to larger changes in normal force and flow rate, relative to the smooth walled case.

\subsection{Effects of the number of corrugation waves}

Effects of the number $n$ of corrugation waves on normal force and flow rate are presented in Figures 8 and 9. Comparison between Figures 8, 4 and 6 indicates that $n$ has similar influence on normal force to the corrugation shape and corrugation amplitude. Thus, the effects of higher $n$ value correspond to those of corrugation shape with larger volume, and of corrugation with larger amplitude. Similar remarks also apply to flow rate, as comparison between Figures 9, 5 and 7 shows.

On the other hand, while trends in the changes (relative to the smooth walled case) are similar, Figures 8 and 9 also indicate that the influence of $n$ is milder than that of corrugation shape, location or amplitude. Thus, as $n$ 


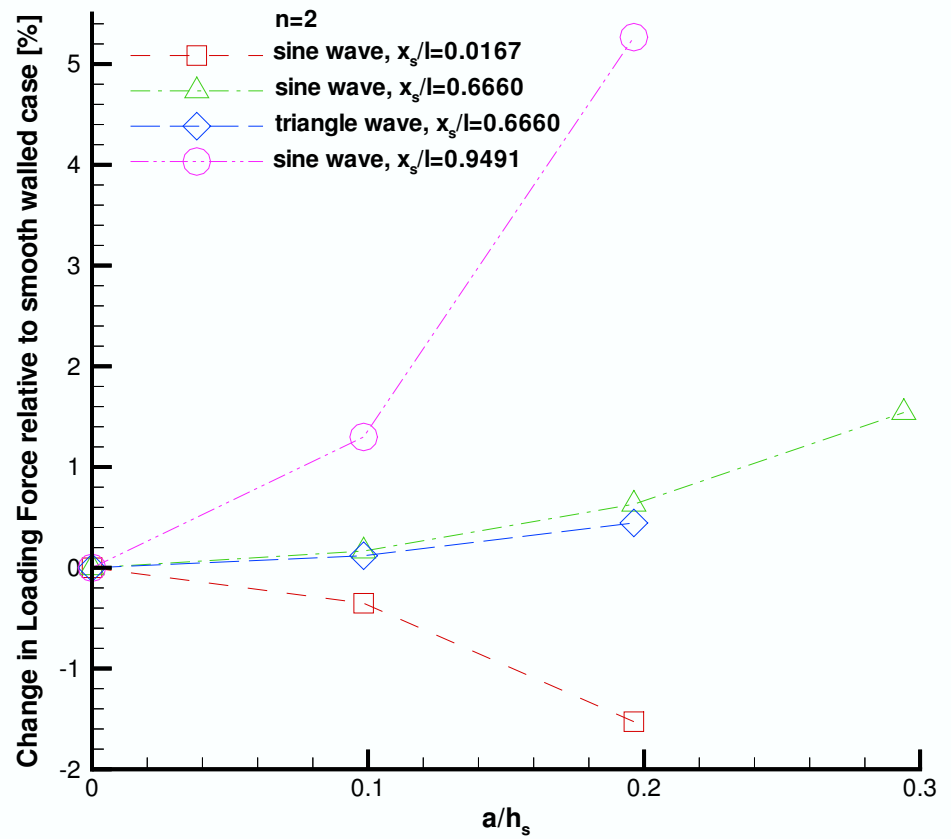

FIGURE 6: Effects of corrugation location and corrugation amplitude on normal load. 


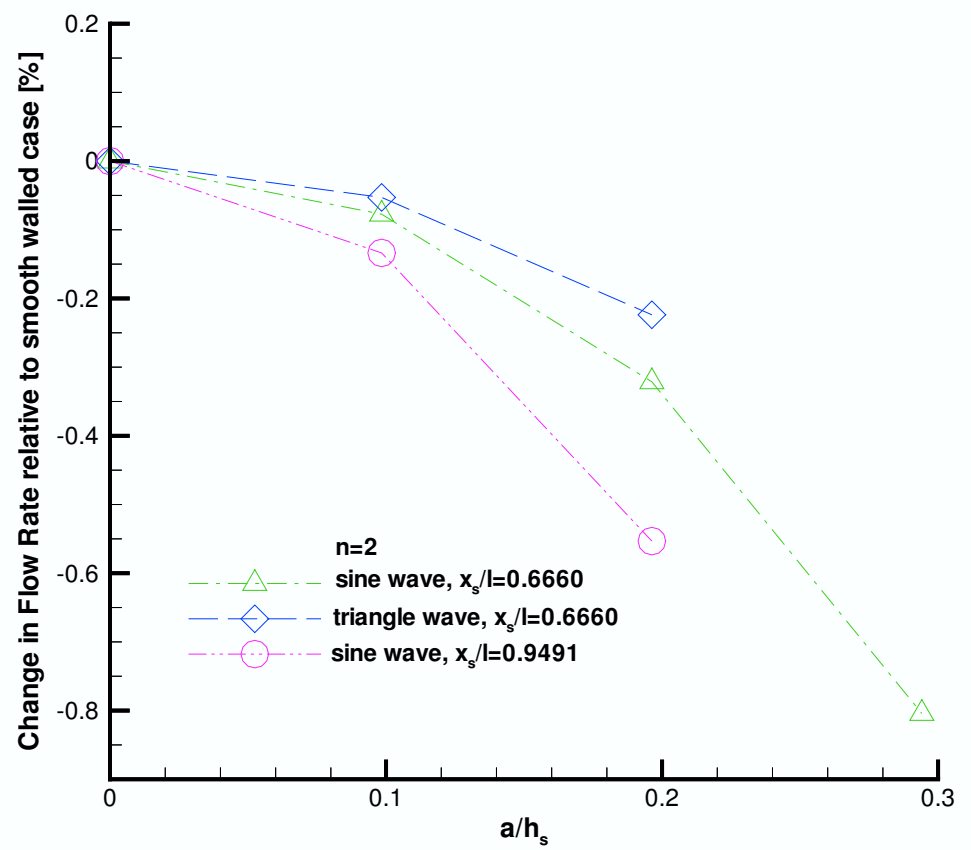

FIGURE 7: Effects of corrugation location and corrugation amplitude on flow rate. 
increases from 1, corresponding changes in the normal force and flow rate are fairly small.

\section{Conclusions}

Influence of corrugation shape, location and the number of corrugation waves (when this number is very small and corrugation in the form of regular waves) on the performance of fixed-incline slider bearings has been investigated numerically, using viscous fluids of a Newtonian type for lubricant and a finite element method.

Of the three corrugation shapes considered, it has been seen that rectangle waves produce largest changes in pressure distribution, normal force and flow rate, relative to the smooth walled case. This is followed by sine waves, whereas triangle waves produce smallest changes. The number of corrugation waves produces similar, but weaker, trends in the changes of normal force and flow rate, to the corrugation volume, when this is used as a parameter characterizing corrugation shape.

It is also seen that normal force increases uniformly, from being less than that of the smooth walled case to being higher, as corrugations are relocated downstream, from being near the gap inlet to being near its exit. On the other hand, flow rate decreases, also uniformly, as corrugations are relocated downstream, toward the gap exit.

\section{References}

[1] G. Bayada and M. Chambat, "New Models in the Theory of the Hydrodynamic Lubrication of Rough Surfaces", ASME Journal of Tribology 110 (1988) 402-407. C1018 


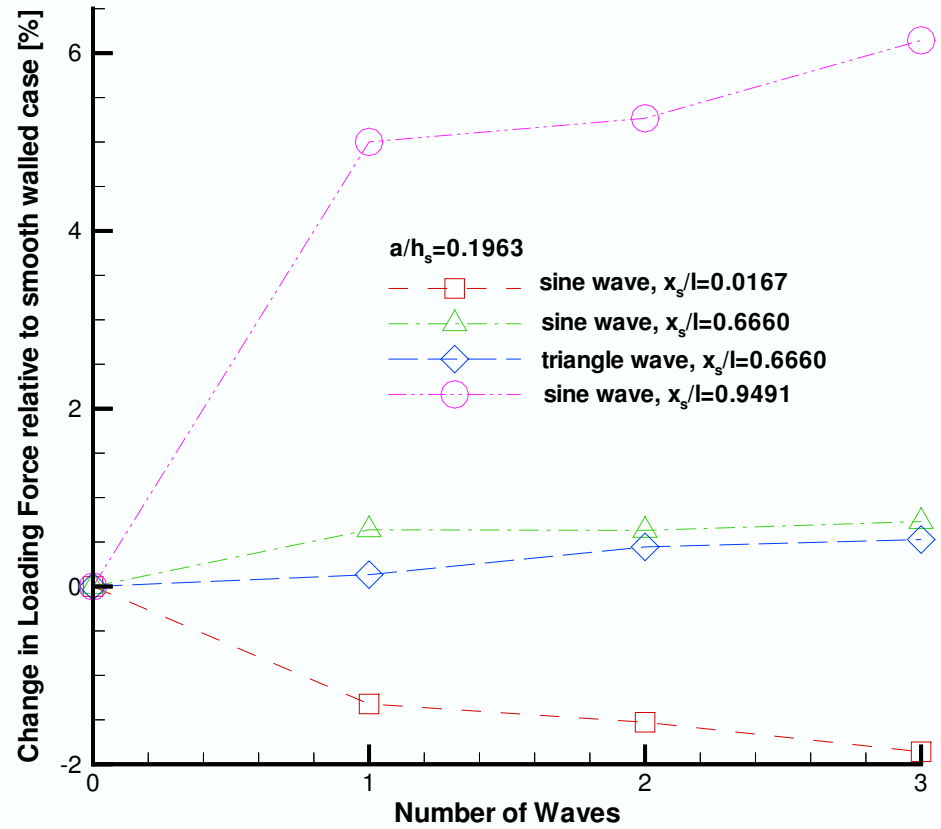

FiguRE 8: Effects of number of corrugation waves and corrugation location on normal load. 


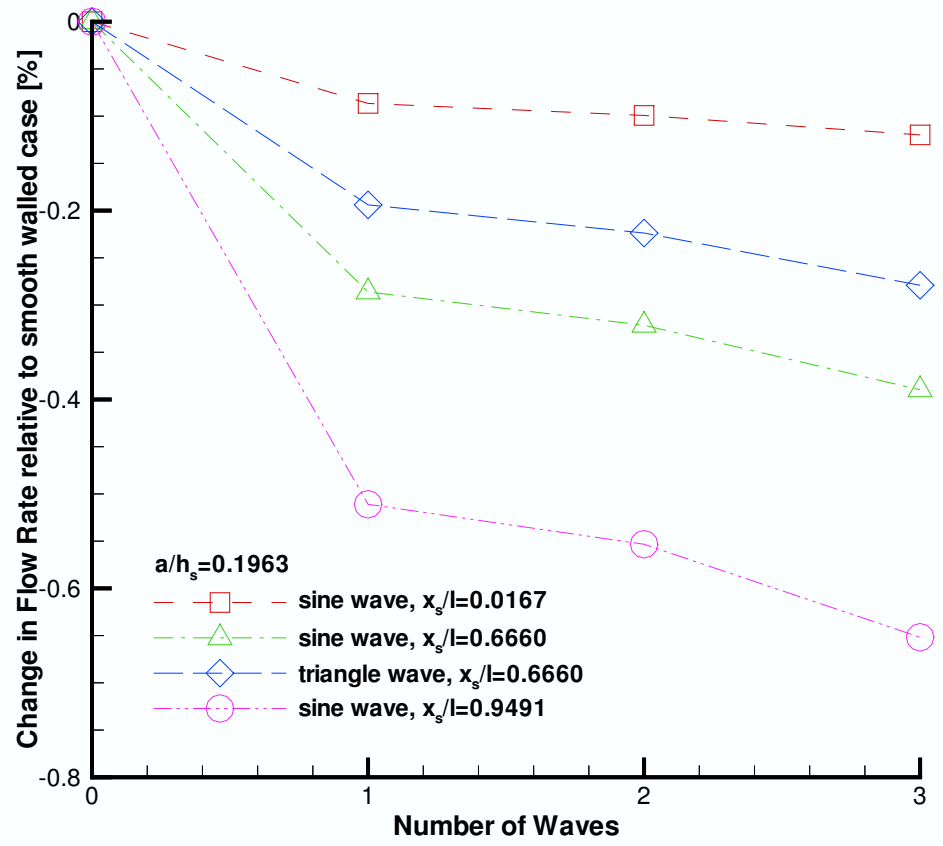

FIGURE 9: Effects of number of corrugation waves and corrugation location on flow rate. 
[2] P. L. Chow and E. A. Saibel, "On the Roughness Effect in Hydrodynamic Lubrication", ASME Journal of Lubrication Technology 100 (1978) 176-179. C1018

[3] H. Christensen and K. Tonder, "The Hydrodynamic Lubrication of Rough Bearing Surfaces of Finite Width", ASME Journal of Lubrication Technology 93(3) (1971) 324-330. C1018, C1019

[4] H. G. Elrod, "Thin-Film Lubrication Theory for Newtonian Fluids with Surfaces Possessing Striated Roughness or Grooving", ASME Journal of Lubrication Technology 95 (1973) 484-489. C1018, C1019

[5] H. G. Elrod, "A General Theory for Laminar Lubrication with Reynolds Roughness", ASME Journal of Lubrication Technology 101 (1979) 8-14. C1018, C1019

[6] R. F. Gans, "On Random Reynolds Roughness", ASME Journal of Tribology 109 (1987) 427-431. C1018, C1019

[7] Y. Mitsuya and S. Fukui, "Stokes Roughness Effects on Hydrodynamic Lubrication. Part 1 - Comparison between Incompressible and Compressible Lubricating Films", ASME Journal of Tribology 108 (1986) 151-158. C1018, C1019

[8] D.-C. Sun and K.-K. Chen, "First Effects of Stokes Roughness on Hydrodynamic Lubrication", ASME Journal of Lubrication Technology 99(1) (1977) 2-9. C1018, C1019

[9] J. W. White, P. E. Raad, A. H. Tabrizi, S. P. Ketkar and P. P. Prabhu, "A Numerical Study of Surface Roughness Effects on Ultra-Thin Gas Films", ASME Journal of Tribology 108 (1986) 171-177. C1018, C1019

[10] J. D. Frêne, D. Nicolas, B. Degueurce, D. Berthe and M. Godet, Hydrodynamic Lubrication - Bearings and Thrust Bearings (Elsevier, Amsterdam, 1997). C1021 
[11] R. B. Bird, W. E. Stewart and E. N. Lightfoot, Transport Phenomena (Wiley, New York, 1960). C1022

[12] B. J. Hamrock, Fundamentals of Fluid Film Lubrication (McGraw-Hill, New York, 1994). C1023, C1024, C1026

[13] B. P. Huynh and R. I. Tanner, "Finite Element Study of Some Flow Problems with Thermally Induced Buoyancy", Proceedings of the 8th Australasian Fluid Mechanics Conference (Newcastle, Australia, 28 November - 2 December, 1983) 8B.1-8B.5. C1023

[14] B. P. Huynh, "A Numerical Investigation of Non-Isothermal Extrusion through Annular Dies", International Journal of Engineering Science 36(2) (1998) 171-188. C1023

[15] B. P. Huynh, "Thermal Effects on Coat Thickness in Wire-Coating Process", Proceedings of the International Conference on Advanced Polymers and Processing, (Yonezawa, Japan, 30 October - 2 November, 2001) 3-9. C1023

[16] P. E. Gill, W. Murray and M. H. Wright, Numerical Linear Algebra and Optimization - Volume 1 (Addison-Wesley, Redwood City, California, 1991). C1024 